\title{
Syndrome-specific deficits of performance and effects of practice on arm movements with deafferentation due to posterior thalamic lesion
}

\author{
Thomas Platz and Karl-Heinz Mauritz
}

\author{
Klinik Berlin, Abteilung für Neurologische Rehabilitation, Freie Universität Berlin, \\ Germany
}

Correspondence to: Dr T Platz, Klinik Berlin, Department of Neurological

Rehabilitation, Free University Berlin, Kladower Damm 223, 14089 Berlin,

Germany

\begin{abstract}
Aiming and tapping movements were analysed repeatedly over a three-week period in a patient who was hemideafferented due to an ischaemic posterior thalamic lesion. Contrasting behaviour observed in six healthy subjects, nine hemiparetic patients and one patient with hemianopic stroke, allowed the determination of behavioural deficits related to deafferentation. Finger tapping was not impaired specifically and did not improve with practice in the deafferented patient. When aiming movements were investigated, accuracy of the first, largely preprogrammed, phase of movement and timing of the late homing-in phase were impaired specifically in the deafferented patient. Practice led to a step-like change in preprogramming amplitude of the ballistic movement component, a gradual improvement of temporal efficiency of the early movement phase and a more marked improvement of the homing-in phase. Qualitatively comparable but quantitatively less marked effects of practice were documented for hemiparetic patients. These results demonstrated that deafferentation affects preprogrammed aspects of movement and those influenced by current control and that motor learning is possible with central deafferentation, even for aspects of performance that are impaired specifically. It is postulated that motor learning was mediated by changes in strategy (motor programming) and improved efficiency of intact motor control processes (visuomotor control).
\end{abstract}

Keywords: Afferent pathways - Thalamus - Learning - Psychomotor performance

\section{INTRODUCTION}

Mott and Sherrington (1895) found that surgical deafferentation of a limb of a monkey led to an unwillingness to use the limb in purposeful action. Subsequently, it was shown repeatedly that deafferentation in monkeys does not abolish the capacity to make purposeful movements but leads to poor coordination and inaccuracy of movements (Munk, 1909; Knapp et al., 1963). In man, impaired proprioception was shown to result in deficits in fine control of movement, failure of accurate postural maintenance (Rothwell et al., 1982; Sanes et al., 1985) as well as deficits in preprogramming of multi-joint aiming movements (Ghez et al., 1990). Positive effects of practice on motor behaviour were reported in animal studies (Munk, 1909; Taub, 1977) but these effects have not been investigated in detail in man.

In this study, deficits of performance of finger tapping and aiming movements were investigated kinematically in a patient with deafferentation due to ischaemic thalamic damage. Comparing the level of performance in this patient with that observed in hemiparetic and hemianopic patients and in healthy subjects revealed behavioural deficits related to deafferentation. Further investigations were undertaken to determine whether motor learning occurred with training in the deafferented patient, which aspects of motor behaviour showed improvement and how the effects of training compared with those demonstrated in hemiparetic patients receiving the same training schedule. 


\section{METHODS}

\section{Subjects}

All subjects were right-handed. A. Patient G, a 70-year-old woman, suffered an ischaemic stroke five weeks prior to the investigation. Areas of the brain affected were in the distribution of the right posterior cerebral artery. The right occipital lobe as well as posterior thalamus, including the ventroposterolateral nuclei (Cranial CT). Clinical examination revealed preserved cognitive abilities, an incomplete left-sided homonymous hemianopia, a severe left-sided hemisensory deficit which affected all somatosensory modalities, including position sense, and a minimal left-sided hemiparesis. B. Patient K, a 60-year-old woman with a cerebro-vascular accident (CVA) in the distribution of the right posterior cerebral artery according to CCT (ischaemic area) and clinical findings (minimal hemiparesis, hemianopia). Patient $\mathrm{K}$ resembled the deafferented patient closely, although somatosensory function and the thalamus (CCT) were not affected. C. Eight hemiparetic patients, six males and two females, with a mean age of 64.9 years $(\mathrm{SD}=5.1)$ developed an ischaemic subcortical stroke in the distribution of the right middle cerebral artery four to eight weeks prior to the investigation. At the time of investigation, all had a minimal left-sided hemiparesis without somatosensory, visuomotor (optic ataxia), attentional (neglect), apraxic or other higher cognitive impairment. D. Six healthy subjects, three males and three females with a mean age of 57.8 years $(\mathrm{SD}=11.3)$.

\section{Motor tasks}

Subjects were seated at a desk. The hand and forearm were rested on the desk in a comfortable position. Fifty fastest repetitive vertical movements (tapping) were performed with the left index finger. In addition, 10 fast and accurate aiming movements of the left arm in a midsagittal plane over a distance of $20 \mathrm{~cm}$ to a target of $5 \mathrm{~mm}$ in diameter were registered and analysed. For motion analysis, an optoelectronic system (SELSPOT II) with two cameras and one (tapping) or two (aiming) infra-red light emitting diodes (IRED LEDs) was used; sample frequency was $100 \mathrm{~Hz}$. Data were lowpass-filtered using a digital Fast-Response-Filter at $20 \%$ of sample frequency. Mean values and standard deviation for the following kinematic parameters were calculated by automatic analysis based on position data and their time derivatives (velocity and acceleration): duration of taps, duration of aiming movements and their components, time before (phase 1) and after maximal deceleration (phase 2), spatial error at the end of aiming movements with respect to the centre of the target (accuracy), and distance from target at time of maximal deceleration (accuracy of phase 1). Values of kinematic parameters were considered pathological if mean values deviated by more than two SDs from the corresponding mean value of the control group of healthy subjects (patients $\mathrm{G}$ and $\mathrm{K}$ ) or if group differences were statistically significant (hemiparetic patients). Syndrome-specificity for deafferentation was assumed if values for patient $G$ differed more than two SDs from the mean value of healthy, hemianopic and hemiparetic control subjects. Using this approach, it was thought that any contributing effect of a minimal hemiparesis or hemianopia in patient $G$ could be deducted, allowing inferences to be made about specific behavioural sequelae due to deafferentation.

\section{Training}

Aiming movements were tested in patient $\mathrm{G}$ on fifteen consecutive weekdays, (excluding weekends), over a period of three weeks. During the first week (days 1 to 5) and the third week (days 15 to 19), the patient did not practise the tasks between recordings on consecutive days. Starting on the last day of the first week (day 5) and during the second week (days 8 to 11) the patient practised the two motor tasks for 20 minutes each day (eight blocks of 2.5 minutes, with alternating tapping and aiming) under supervision. Analysis of motor tasks during the second week was always performed at least 21 hours after the last training session. Behavioural changes could then be considered to reflect motor learning rather than changes in performance. Time-series analysis included autocorrelation function (ACF), crosscorrelation function, as well as linear regression model $($ LRM $)$ (kinematic variable $=$ $\mathrm{a}+\mathrm{b}^{*}$ day) (McClearly and Welsh, 1992). The eight patients with minimal hemiparesis received the same practice schedule and were assessed kinematically twice before the period of daily training (corresponding to days 2 and 4 of the schedule for patient $\mathrm{G}$ ). They were also assessed kinematically one day after the last day of training (corresponding to day 12 of the schedule for patient $G)$.

\section{RESULTS}

\section{Baseline performance}

The duration of taps was prolonged in the deafferented patient G. Durations of the first and second phase of the aiming movement were prolonged in patient $G$, the hemiparetic patients and the hemianopic patient (patient $\mathrm{K}$ ). However, movement duration and time after maximal deceleration (phase 2) showed a syndrome-specific marked prolongation in patient $\mathrm{G}$. In addition, the distance from target at time 
TABLE I. Kinematic parameters for tapping and aiming movements (left arm) at baseline for a deafferented patient and control subjects

\begin{tabular}{|c|c|c|c|c|c|c|}
\hline & \multicolumn{5}{|c|}{ Aiming movements } & \multirow{2}{*}{$\begin{array}{l}\text { Tapping } \\
\text { Duration (s) }\end{array}$} \\
\hline & Duration (s) & Phase 1 (s) & Phase 2 (s) & Ph1-Acc $(\mathrm{mm})$ & Acc $(\mathrm{mm})$ & \\
\hline \multirow{2}{*}{$\begin{array}{l}\text { Pat. G, f, } 70 \text { yrs. } \\
\text { (Deafferentation) } \\
\text { Pat. K, f, } 60 \text { yrs. } \\
\text { (Hemianopia) }\end{array}$} & $\underline{2.05}(0.36)$ & $0.75(0.14)$ & $1.30(0.42)$ & $\underline{87}(19)$ & $11(6)$ & $0.45(0.07)$ \\
\hline & $0.85(0.17)$ & $0.42(0.02)$ & $0.43(0.17)$ & $25(6)$ & $5(4)$ & $0.25(0.04)$ \\
\hline \multirow{2}{*}{$\begin{array}{l}\text { Hemiparetic pts. } \\
(\mathrm{N}=8,2 \mathrm{f}, 6 \mathrm{~m}) \\
\text { Healthy subjects } \\
(\mathrm{N}=6,3 \mathrm{f}, 3 \mathrm{~m})\end{array}$} & $0.96^{*}(0.23)$ & $0.62^{*}(0.10)$ & $0.34^{+}(0.17)$ & $28(9)$ & $6(6)$ & $0.34(0.13)$ \\
\hline & $0.69(0.14)$ & $0.49(0.11)$ & $0.20(0.09)$ & $22(8)$ & $9(6)$ & $0.28(0.06)$ \\
\hline
\end{tabular}

Phase 1: duration of movement phase until maximal deceleration; phase 2: duration of movement phase after maximal deceleration; Ph1-Acc: distance from target (accuracy) at time of maximal deceleration (end of phase 1); Acc: distance from centre of target (accuracy) at the end of movement. Figures are mean or grand mean (SD).

${ }^{*} p<0.05{ }^{+} p<0.10$ (U test)

Scores are printed bold, if they deviate by more than 2 SD from the mean of healthy control subjects for patients $G$ and $\mathrm{K}$ or if group means are statistically significantly different; underlined scores are thought to reflect syndromespecific changes.

of maximal deceleration was increased in patient $\mathrm{G}$. The accuracy of the first phase of movement was thus reduced in this patient. These results are shown in Table 1.

\section{Effects of practice}

Concerning duration of taps, ACF and estimation of $\operatorname{LRM}(b=-0.001, p=0.61)$ did not show a trend over the three-week period of observation for patient $G$, while the mildly hemiparetic patients showed, on average, some improvement. The change in duration of taps from the last baseline measurement to assessment after training showed mean values of $-0.07 \mathrm{~s}, \mathrm{SD}=0.08$. The corresponding value for patient $\mathrm{G}$ was $-0.02 \mathrm{~s}$.

However, kinematic parameters for the aiming movement showed considerable evidence of change over time in patient $\mathrm{G}$ as determined by ACF and LRM (Fig. 1). Duration of both the first and second movement phase of aiming movements tended to decrease during the first two weeks of observation $\left(\mathrm{LRM}_{\text {days 1-12: }}:\right.$ phase $1 \mathrm{~b}=-0.019, p<0.001$; phase 2 $\mathrm{b}=-0.06, p<0.001$; compare Fig. 1, A and B) and stabilized during the third week. Calculation of coefficients of the LRM indicate that the trend was three times greater for the second, compared with the first movement phase. Accuracy of the first movement phase improved in a step-wise manner after the first training session (compare days 5 and 8 in Fig. 1C). This parameter was fairly stable before and after the first training session. The difference between days 5 and 8 was $-31 \mathrm{~mm}$ whilst the difference for all other days had a mean value of $-1 \mathrm{~mm}(\mathrm{SD}=14)$. However, temporal kinematic parameters did not change between days 5 and 8 . Accuracy at the target showed day to day variations, but ACF and LRM (b $=0.008, p=0.45)$ indicated no overall trend across weeks. Mildly hemiparetic patients showed, on average, qualitatively similar but quantitatively less marked changes after training. Changes in parameters of the aiming movement in hemiparetic patients are shown in Fig. 1, A, B and $\mathrm{C}$. Changes between the last baseline measurement and assessment after training are: duration of phase 1 , mean $-0.01 \mathrm{~s}, \mathrm{SD}=0.13$ (compared with $-0.07 \mathrm{~s}$ for patient $\mathrm{G}$ ); duration of phase 2 , mean $-0.08 \mathrm{~s}$, $\mathrm{SD}=0.16$ (compared with $-0.12 \mathrm{~s}$ for patient $\mathrm{G}$ ); accuracy of phase 1 , mean $-9 \mathrm{~mm}, \mathrm{SD}=19$ (compared with $-37 \mathrm{~mm}$ for patient $\mathrm{G})$.

\section{DISCUSSION}

Deficits of motor behaviour which were found in the deafferented patient, but were not present to a similar degree in healthy, hemiparetic or hemianopic control subjects, were thought to be related to deafferentation. The specific impairment of aiming movements is, presumably, due to their higher demand on processing somatosensory information in comparison with tapping. The reduced distance covered by the early, largely preplanned movement phase of the aiming movement suggests an alteration of an aspect of preprogrammed movement. The disproportionally increased duration of the second phase of movement 

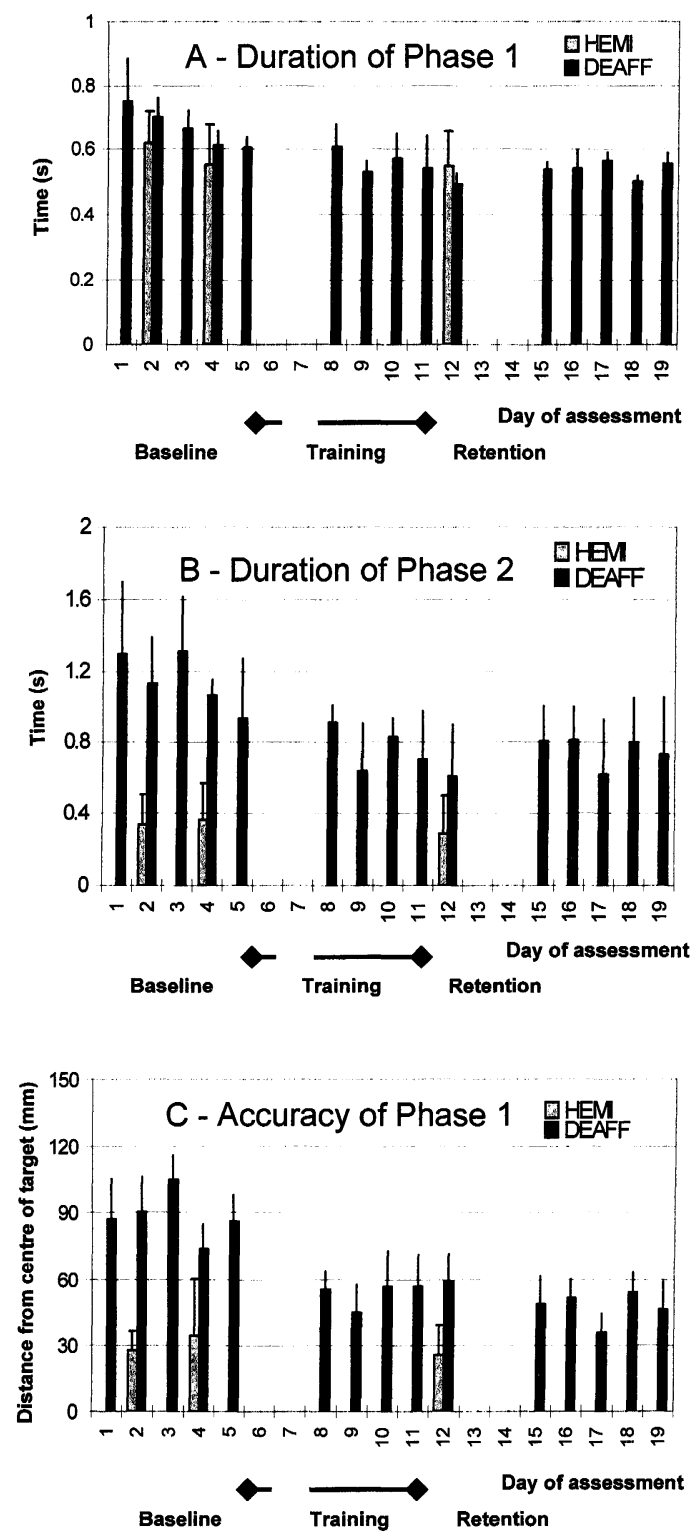

FIG. 1. Kinematic analysis of aiming movements in a deafferented patient and eight hemiparetic patients. Kinematic analysis of standardized aiming movements in a deafferented patient was performed on successive weekdays (days 1 to 5,8 to 12 and 15 to 19 ); training was provided on day 5 and on days 8 to 11 . Eight hemiparetic patients were investigated twice before training (days 2 and 4) and once after training (day 12). Bars denote mean values of 10 discrete aiming movements each day for the deafferented patient or grand mean values for hemiparetic patients, error bars $+/-1$ SD. Figure $1 \mathrm{~A}$ shows duration of the first of two movement components, the time before maximal deceleration (phase 1). Duration of the second component after maximal deceleration (phase 2) is shown in Fig. 1B. Figure 1C shows the distance from the target at time of maximal deceleration $(\mathrm{mm})$ which reflects the spatial accuracy of the first movement phase. was only partially due to the longer distance covered by that phase and gives evidence for a reduced temporal efficiency of processes involved in this phase of movement. Prolongation of the second component of movement has been described for a reaching and grasping task with deafferented patients with either medullary lemniscal or cortical parietal damage (Jeannerod, 1990), indicating that transfer of somatosensory information through the parietal cortex is important for the control mechanisms of the homingin phase of goal-directed movements. It has also been shown that both somatosensory and visual information are relevant for accuracy achieved during the homingin phase and that visuomotor control can partially compensate for somatosensory deficits (Carlton, 1980; Beaubaton and Hay, 1986; Jeannerod, 1990). The altered kinematic characteristics found in the aiming movement which is under investigation could reflect a situation where the deafferented patient, whose parietal cortex is deprived of somatosensory lemniscal input by thalamic damage, deliberately uses more space for the homing-in phase (preprogramming) and needs more time for compensatory visuomotor control during that phase in order to achieve the required accuracy.

For the deafferented patient, different aspects of motor learning, viewed here as lasting changes in motor behaviour, could be demonstrated for the aiming movements which were specifically impaired, while no clear behavioural changes were found for the tapping task. The first session of blocked practice led to a step-like improvement in the distance covered during the first movement phase, independent of temporal changes, presumably reflecting a change of movement strategy for preprogrammed spatial characteristics of that movement phase. A gradual decrease in duration of both the first and second movement phase was observed. These changes cannot be accounted for by proportional changes of the distance moved during each phase or overall change in terminal accuracy. Therefore, the changes reflect increased temporal efficiency of the first, largely ballistic, movement component and alterations of sensorimotor control, with effects on visuomotor control predominating, during the homing-in phase of aiming movements. The effect was three times greater for the late movement phase, indicating a different pattern of learning for each phase. With mildly hemiparetic patients, changes which were quantitatively less but were of a similar qualitative pattern emerged after the practice sessions. Quantitatively smaller effects of training for the first movement phase, in comparison with the second movement phase, or for hemiparetic patients, in com- 
parison with the deafferented patient, could be related to floor effects and do not, necessarily, imply an impairment of motor learning (Schmidt, 1988). The deafferented patient showed no evidence of impaired motor learning, even for aspects of motor behaviour which were specifically impaired. It is suggested that largely intact processes, which are not crucially dependent on somatosensory information and rely on intact frontal motor and parietal association areas at cortical level, were responsible for motor learning in the deafferented patient. Adjusted preprogramming leads to change of spatial coverage for the first movement phase, improved ballistic motor behaviour leads to a reduction in the duration of the first movement phase and improved visuomotor control leads to a reduction in the duration of the second movement phase.

In summary, specific deficits of performance were shown for aiming movements in the centrally-deafferented patient who was under investigation. Subcomponent analysis of aiming movements favours the notion of impaired sensorimotor control during the homing-in phase, leading to strategic adjustment of aspects of preprogrammed movement. Motor learning is possible with deafferentation, as demonstrated in these studies of aiming movement. This is, presumably, based on changes in movement strategy and the efficiency of intact control mechanisms. Such mechanisms are those responsible for the execution of the early, largely ballistic, movement phase as well as those responsible for visuomotor control during the homing in phase.

\section{Acknowledgements}

The contribution of medical students, S Bock and K Prass, with kinematic motion analysis and of occupational therapist, C Pinkowski, with training of patients is gratefully acknowledged. This work was partially conducted while Thomas Platz was in receipt of a Foedor-Lynen fellowship awarded by the Alexander von Humboldt-Stiftung and was supported by BMBF grant $01 \mathrm{~K} 095168$.

\section{REFERENCES}

Beaubaton D and Hay L (1986) Contribution of visual information to feedforward and feedback processes in rapid pointing movements. Human Movement Science $\mathbf{5}$, 19-34.

Carlton LG (1980) Movement control characteristics of aiming responses. Ergonomics 23, 1019-1032.

Ghez C, Gordon J, Ghilardi MF, Christakos CN and Cooper SE (1990) Roles of proprioceptive input in the programming of arm trajectories. Cold Spring Harbor Symposia on Quantitative Biology 15, 837-847.

Jeannerod M (1990) The neural and behavioural organization of goal-directed movements. Clarendon Press, Oxford.

Knapp HD, Taub E and Berman AH (1963) Movements in monkeys with deafferented forelimbs. Experimental Neurology 7, 305-315.

McClearly R and Welsh W (1992) Philosophical and statistical foundations of time-series experiments. In: Singlecase Research Design and Analysis (Eds TR Kratochwill and JR Levin), pp.41-91. Lawrence Erlbaum Associates, Hillsdale.

Mott FW and Sherrington CS (1895) Experiments upon the influence of sensory nerves upon movement and nutrition of the limbs. Proceedings, Royal Society (London B) 57, 481-488.

Munk H (1909) Über die Folgen des Sensibilitätsverlustes der Extremität für deren Motilität. Kap XIII. In: Über die Funktionen von Hirn und Rückenmark, Gesammelte Mitteilungen (Ed H Munk), pp. 247-285. Hirschwald, Berlin.

Rothwell JC, Traub MM, Day BL, Obeso JA, Thomas PK and Marsden CD (1982) Manual motor performance in a deafferented man. Brain 105, 515-542.

Sanes JN, Mauritz K-H, Dalakas MC and Evarts EV (1985) Motor control in humans with large-fibre sensory neuropathy. Human Neurobiology 4, 101-114.

Schmidt RA (1988) Motor learning: fundamental concepts and research methods. In: Motor control and learning (Ed RA Schmidt), pp. 345-376. Human Kinetics, Champaign.

Taub E (1977) Movement in nonhuman primates deprived of somatosensory feedback. In: Exercise and Sports Sciences Reviews, Vol 4 (Ed KF Keogh), pp. 335-374. Journal Publishing Affiliates, Santa Barbara.

(Received 24 July 1995; accepted 18 January 1997). 


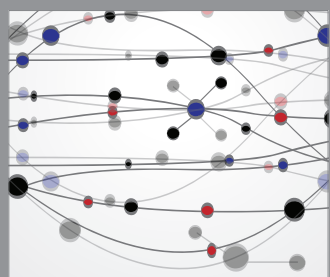

The Scientific World Journal
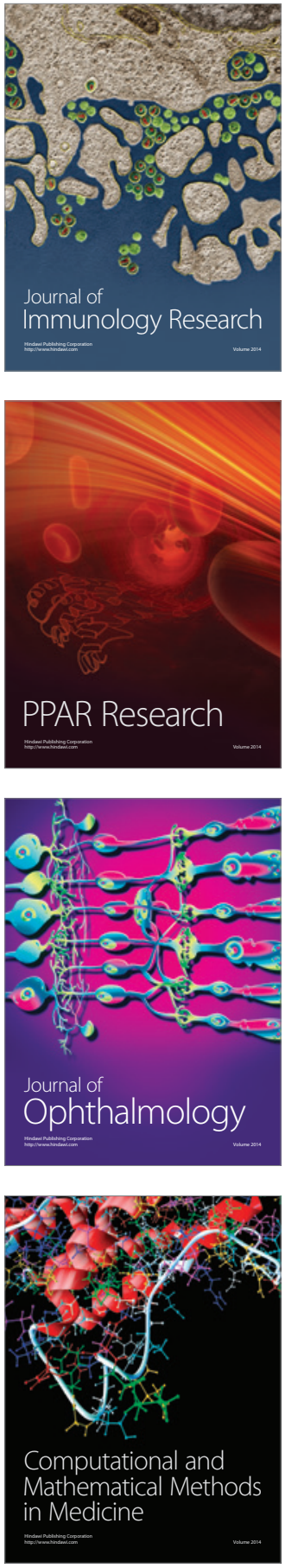

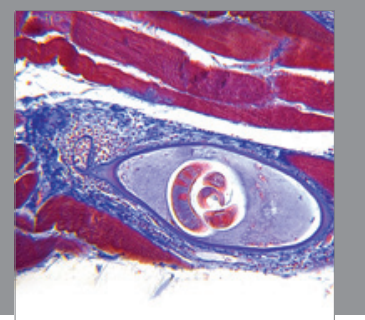

Gastroenterology

Research and Practice
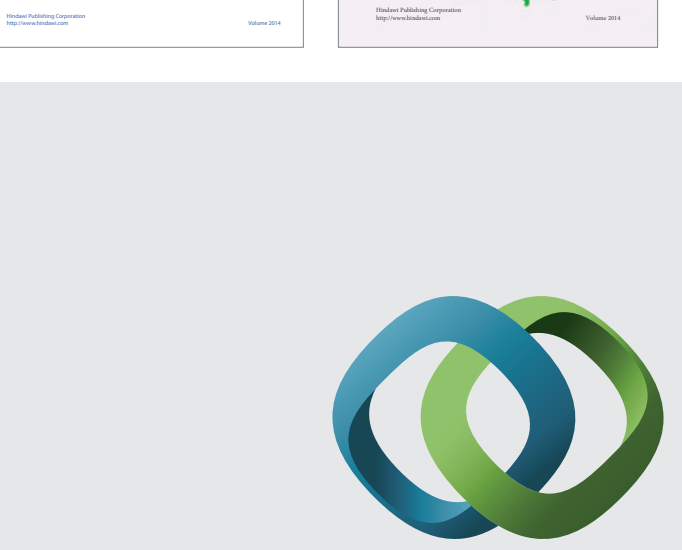

\section{Hindawi}

Submit your manuscripts at

http://www.hindawi.com
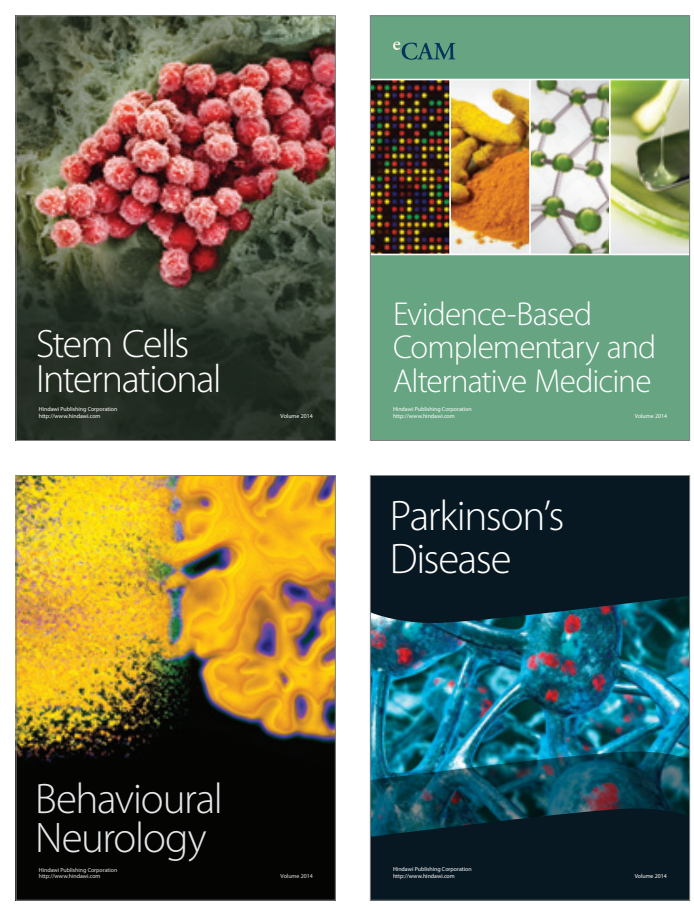

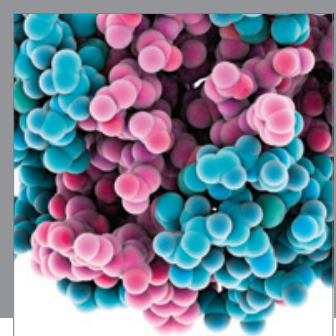

Journal of
Diabetes Research

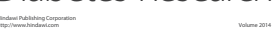

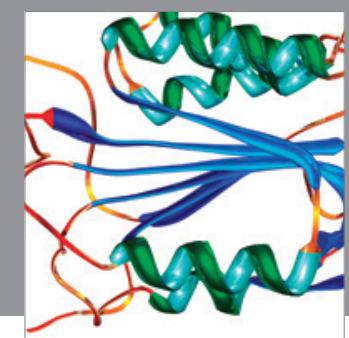

Disease Markers
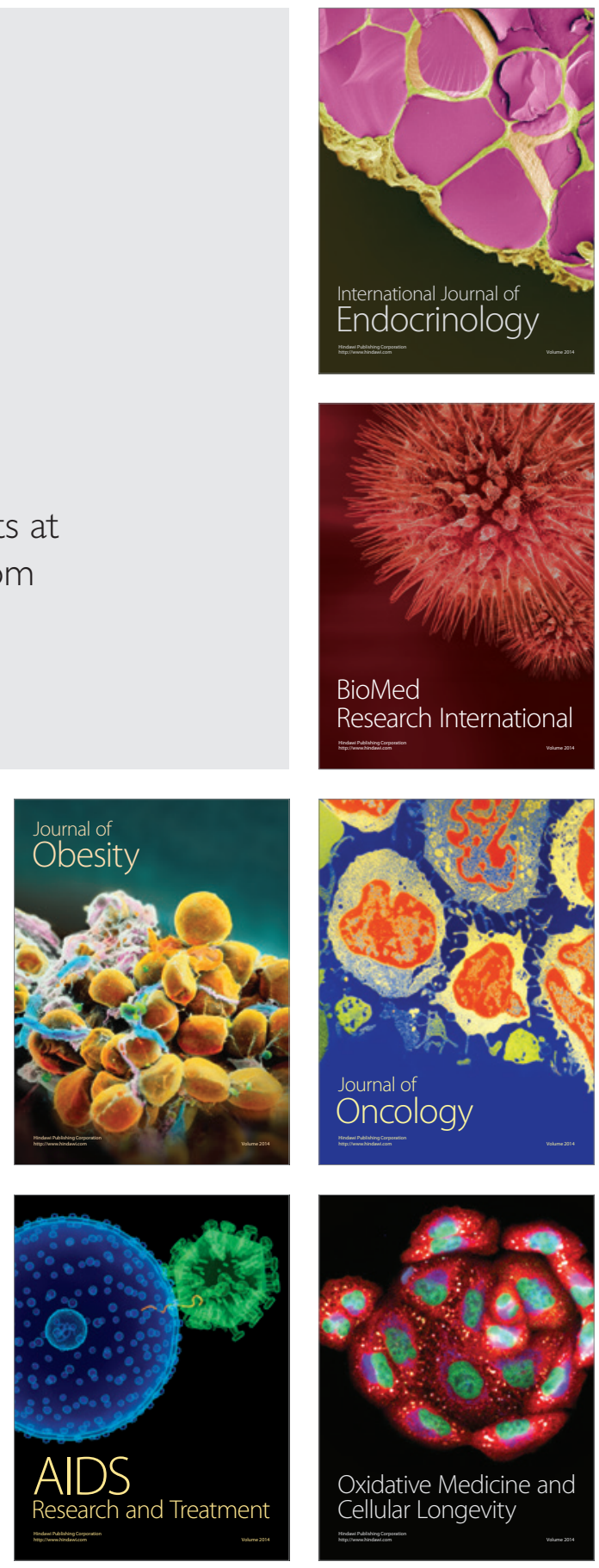